\title{
STILL'S DISEASE IN ADULTHOOD
}

Elisa Fernandes de Melo ${ }^{1}$, Raylane Shellyda de Almeida Anate ${ }^{1, *}$, Aline Gimenez Guerra ${ }^{1}$, Thais Perrone El Saman ${ }^{1}$, Isabel Penteado Sitolini ${ }^{1}$, Marina Fernandez Florençano ${ }^{1}$

1.Universidade de Taubaté, Taubaté (SP), Brazil.

${ }^{*}$ Corresponding author: raymed87@gmail.com

\section{BACKGROUND}

Adult Still's disease is a rare rheumatologic disease characterized by high fever, skin rash and arthritis. The etiology is still unknown. The disease typically affects young adults, occurring before the age of 35 in $76 \%$ of cases. Weight loss is reported in 50 to $60 \%$ of patients and can be dramatic and rapid and correlated with inflammatory activity, measured by hemoglobin and albumin drop. The most common fever pattern is that of febrile peaks, manifesting once to twice a day and reaching $40^{\circ} \mathrm{C}$. One of the important characteristics of this fever is the occurrence of febrile peaks always at the same time, especially at night, accompanied by profuse sweating. The exanthem is characterized by being evanescent, salmon-colored, appearing frequently during febrile peaks and lasting a few hours. Arthritis is usually the last manifestation to appear, at the beginning there is predominance of a picture of arthralgia, myalgia, morning stiffness and less frequently of synovitis. Arthritis can be oligoarticular, but, most of the time, it is additive, affecting large and small joints. Lymphadenomegaly, splenomegaly and hepatomegaly are very frequent at the onset of the disease, occurring reflect tissue infiltration by inflammatory cells and increased immunological activity in the reticuloendothelial system. Although adult Still's disease has several characteristics of a systemic inflammatory disease, it has neither a positive rheumatoid factor nor antinuclear antibody (ANA). Most patients have neutrophilic leukocytosis with leukocytes around 12,500 to 40,000 cells per mm 3 . Extreme leucopenia or leukocytosis are rare. Elevation of acute phase reagents is frequent, including ESR, CRP, serum amyloid A, complement, haptoglobin and serum ferritin (extreme elevations above 40,000 deserve differential diagnoses with hemochromatosis, neoplasms, pancreatitis or other inflammatory diseases, such as rheumatoid arthritis or systemic lupus erythematous). For the diagnosis of adult Still's disease is necessarily a diagnosis of exclusion. The need to rule out infectious, neoplastic and other rheumatologic diseases often slows the diagnosis. The finding of reactional leukocytosis usually directs the diagnosis to adult Still's disease. The recommended treatment is similar to that of rheumatoid arthritis. Indomethacin is the anti-inflammatory of choice, with good action also against fever. Low-dose oral corticosteroids and DMARDs are indicated for joint manifestations. The prognosis of adult Still's disease is good. Around $20 \%$ of patients have a monocyclic disease lasting 4 to 12 months.

\section{CASE REPORT}

Patient, female, 77 years old, has a history of leukopenia and anemia in 1999, 3 years earlier anemic condition was found associated with afternoon fever $\left(38-40^{\circ} \mathrm{C}\right)$, odynophagia, weight loss $(10 \mathrm{~kg})$, symmetrical joint pain with local edema, mainly in proximal fists and interphalangeal with morning stiffness of about $2 \mathrm{~h}$, with the appearance of desquamative erythematous, pruriginous skin lesions, in the region of the back and neck, which disappeared spontaneously after 2 months. The whole condition improved significantly after the onset of corticosteroid therapy in 2017. After discontinuation of corticosteroid therapy (prednisolone), he returned with apathy, hyporexia, fever $\left(39.5-40^{\circ} \mathrm{C}\right.$ ) constant, hair loss, diffuse joint pain so movement and an episode of important odynophagia in 07/09/2019. She was referred to the service of the Municipal University Hospital of Taubaté on 12/09/2019. Investigation was initiated with the rheumatology team, where an important increase in ferritin $(24,846)$ diagnosed then with late-onset adult Still's disease, where the use of prednisone $0.5 \mathrm{mg} / \mathrm{kg} /$ day began. Patient remains in good general condition, stable clinically and hemodynamically, in outpatient follow-up with rheumatology.

\section{CONCLUSION}

Adult Still's disease should always be considered in the differential diagnosis approach, continued physical observation of patients with prolonged febrile syndromes may reveal crucial signs leading to diagnosis, especially associated with cutaneousarticular picture. It should be emphasized that this diagnosis is always exclusionary and it is necessary to investigate infectious and neoplastic diseases, among others. 